selbst, etwa an die Bundesterrasse, oder an die Engehalbinsel, mit der Stadt und dem Münsterturm im Vordergrund, wenn die Berge im Mittagsglanz leuchten oder im Alpenglühen stehen - wie viele sind dann gebannt von diesem Anblick!

Noch weiter! Wir gedenken des Blickes vom Jura über das Mittelland mit seinem Mosaik von Äckern, Wiesen und Wäldern vom Chaumont, Chasseral, dem Weißenstein zum Kranz der Alpen mit der zentralen Gestalt der Jungfrau. Ja, über den Jura hinweg vom Gebweiler Belchen in den Vogesen ist die Jungfrau zu sehen.

Bei großen Teilen des Alpenpanoramas, das vom Mittelland aus gesehen werden kann, werden die meisten Betrachter nur ganze Gebirgsgruppen benennen können, häufig sind es nur einfach «die Alpen». Die Berneralpen mit ihren ausgeprägt individuell profilierten Gestalten machen indessen eine Ausnahme und wenn auch die Namenkenntnis der Betrachter als sehr gering zu veranschlagen ist, so kennen doch die meisten den Namen der Jungfrau.

Die Wirkungen dieses Berges sind nicht nur weitreichend, sondern auch mannigfaltiger Art. Sie sind auch wirtschaftlich nicht zu unterschätzen, wenn wir an den Fremdenverkehr denken. Dies findet bei manchem Hotel Ausdruck, das Jungfrau oder Jungfraublick heißt. Über dieses noch in Franken und Besucherzahlen Meßbare hinaus reicht die Bedeutung ins Unmeßbare. Durch Jahre trugen die eidgenössischen Abstimmungsanschläge als zierende Kopfzeichnung die Jungfrau. Denn die Jungfrau, die zu gewissen Tageszeiten ein Schattenkreuz trägt, ist weit über das Ästhetische und über das Bergerlebnis des Einzelnen hinaus zu einem eidgenössischen Symbol geworden.

So zeigt das Beispiel der Jungfrau, daß der Einfluß eines Berges weit über seinen Standort ausstrahlen kann und die geographische, die landschaftliche Bedeutung einer solchen Gestalt nur erfaßt wird, wenn neben die rein naturwissenschaftlich-objektive Beschreibung die ästhetisch-psychologische Würdigung tritt.

Michel, H.: Die Jungfrau, Berner Heimatbücher Nr. 25 Bern - Lucerna, R.: Matterhorn. Peterm. geogr. Mitteilungen 22, 1936. - Niggli, P.: Schulung und Naturerkenntnis. Zürich 1945. - Hartog, J. M. Muztag Tower. Die Alpen 1957 - Boss, V. Lütschinentäler. Berner Wanderbuch 6. Bern 1959.

\title{
ZUR BEWÄSSERUNG DER POEBENE
}

\author{
Walter Nelz
}

\section{EIN LEITUN G}

Bewässerungsanlagen sind wichtige Kulturlandschaftselemente in weiten und geschichtlich bedeutungsvollen Gebieten der Erde. Geographen und Geschichtsforscher kennzeichneten die orientalischen Kulturen vom Nil bis zum Hoangho als Fluß-und Bewässerungskulturen (potamisch) und stellten ihnen die europäischen Meerkulturen (als thalassisch und ozeanisch) gegenüber, wie z. B. ERnst KAPP. KARL MARX und andere verstanden als grundlegendes Element der «asiatischen» Produktionsweise den großen Bewässerungslandbau, der in der Sumpfreiskultur eindrücklich in die Erscheinung tritt.

Nach diesen Schriftstellern entstand und entwickelte sich anfangs die territorialstaatliche Zivilisation in den Flußtälern des Orients. Die Nutzung des Wassers großer Ströme erforderte soziale Kooperation und war deshalb ein organisierender, staatsbildender Faktor ersten Ranges. In unserer Zeit aktualisieren sich die alten Beziehungen zwischen Morgen- und Abendland auf neue Weise. Die orientalischen Kulturen beanspruchen deshalb unsere Aufmerksamkeit in steigendem Maße. Es sei in diesem Zusammenhang nur auf die Schriften des Soziologen K. A WitTFOGEL verwiesen. In «Wirtschaft und Gesellschaft Chinas» (Leipzig 1931) untersuchte er sowohl die Funktion der Bewässerung im landwirtschaftlichen Produktionsprozeß Chinas, als 


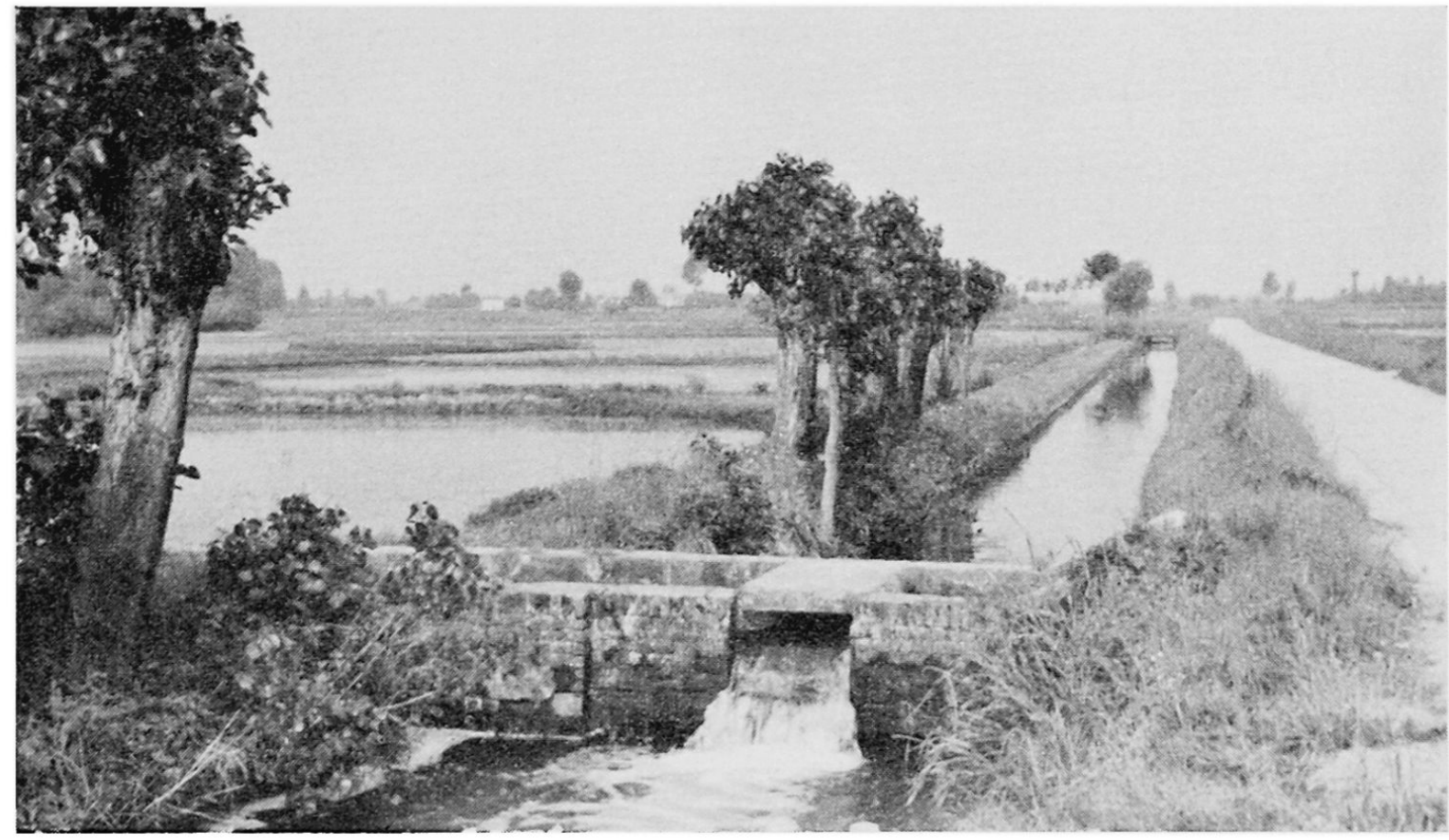

Reisfelder mit Bewässerungskanälen bei Vercelli

(Photo W. Nelz)

auch die Bedeutung des Staates als übergreifendes Organ der Agrarproduktion, d. h. als Organisator der großen Wasserbauten. In «Oriental Despotism» (New York 1957) behandelt er das Problem der «asiatischen»Produktionsweise in allgemeiner und vergleichender Form und diskutiert ausführlich Naturbedingtheit, Wirtschaftsweise, Staatsmacht, Verbreitung, Besitz- und Klassenverhältnisse der Wasserbau-Gesellschaft, sowie die Geschichte der Theorie der «asiatischen»Produktionsweise. Insbesondere diese Werke regten mich dazu an, einmal eine große Bewässerungslandschaft, wenn möglich mit Reisbau, genauer kennen zu lernen. Meine Wahl fiel auf die nahe Poebene. Die auf zwei Exkursionen gewonnenen Beobachtungen seien im folgenden zusammengefaßt wiedergegeben.

Wer im Frühling oder Sommer von Mailand nach Pavia, Vercelli oder Novara reist, dem fallen die vielen Wasserläufe auf, die die weite Ebene durchziehen. Man sieht das Wasser in zahlreichen Kanälen durch die ebene Landschaft rinnen, sieht es die weiten Flächen der von niederen Deichen und Baumreihen umgrenzten Reisfelder schuhtief bedecken, hört die Frösche quaken und staunt über die Vielfalt sinnvoller Wasserbauten: die Verteilung des Wassers aus großen in kleine Kanäle, die Überführung von Kanälen auf Kanalbrücken über Straßen, Flüsse und andere Kanäle, die Unterführung in Dückern (Sifonen) unter Straßen, Flüssen und Kanälen, Kanalüberquerungen von Taleinschnitten auf Aquädukten, die Kanaltunnels durch Hügel, der Durchfluß des Wassers durch leicht gestufte Reisfelder und seine Abführung in Sammel- und Abflußkanäle usw. Das Wasser der Alpenflüsse wird in einem sinnvoll nivellierten und kommunizierenden Gefäßsystem fast lückenlos über die weite Ebene verbreitet und was davon übrig bleibt, in das natürliche Abflußsystem zurückgeleitet. Die künstlich überschwemmten Reisfelder um Vercelli gleichen im Frühling einem riesigen, von Straßen, Wegen, Höfen und Dörfern, Dämmen, Kanälen und Baumreihen durchmusterten See. Man erlebt eine Wasserbaulandschaft von hoher Entwicklung und hoher Intensität. Der Hauptzweck der Wasserbauten ist eindeutig die agrikole Befeuchtung des Landes. Stellenweise sind in geeigneter Lage industrielle Anlagen wie Mühlen und Kraftwerke eingebaut. Einige Kanäle dienen bescheidener Lastschiffahrt, wie z. B. der im Sand- und Kieshafen von Mailand endende Naviglio Grande dem gelegentlichen Sand-, Kies- und Steintransport. Die Steine für den Dom sind auf ihm vom Langensee herüber transportiert worden.

Es ist verständlich, daß mich das optische Erlebnis erst recht anregte, einen Überblick über die großen Bewässerungsanlagen der Poebene zu gewinnen. Bei meinen Erkundigungen erhielt ich in Zürich eine Empfehlung an den Direktor des Ufficio Idrografico del Po in Mailand, Ugo RAFFA. Ich begab mich daraufhin im Oktober 1959 ein zweites Mal in die Poebene. In Mailand sah ich nun die Bewässerungskarten der Lombardei und des Piemont 1: 10000 aus den Jahren 1929 und 1930, sowie verschiedene die Bewässerung dieser Gebiete behandelnde Schriften. Herr Raffa zeigte mir anderntags Fontanili westlich von Mailand, sowie die Wasserbauten am Tessin unterhalb von Sesto Calende: 1. das vom Consorzio del Ticino betriebene Regulierwehr des Langensees; 2. die Staustufe bei Porto della Torre mit der Fassung des 
neuen Kanals Regina Elena, der der Ergänzung des Cavour-Systems östlich der Sesia dient; 3. die Fassung des Villoresi-Kanals und des Industriekanals von Vizzola bei Pamperduto, der, nach dem er mehrere Kraftwerke antrieb, den Naviglio Grande speist. In Novara besuchte ich anderntags die Zentralverwaltung der größten Bewässerungsgenossenschaft Italiens, derjenigen für das Gebiet zwischen Sesia und Tessin (Associazione Irrigazione Est Sesia), wo ich wertvolle Aufschlüsse und die Publikationen der Gesellschaft erhielt. In Vercelli besuchte ich die Zentrale der Bewässerungsgenossenschaft des Gebietes zwischen Sesia und Dora Baltea, sowie die Stazione Sperimentale di risicoltura e delle coltivazioni irrigue, wo mir der Reisspezialist Prof. PIAcco Auskunft erteilte; in Turin desgleichen die Zentralverwaltung der Canali Demaniali d'Irrigazione Cavour. Generaldirektor Zanaboni empfahl mich dem Distriktsdirektor Stasia in Chivasso, der mir die Fassung des Cavour-Kanals zeigte, sowie die Kanal- und Pumpanlagen im Gebiet der Dora Baltea und des Cavour-Kanals zwischen Chivasso, Ivrea und Santhià. Die reifen Reisfelder leuchteten goldgelb in der Oktobersonne. Die Ernte war in vollem Gange. Schließlich besuchte ich auf dem Rückweg in Mailand noch die Verwaltung des Villoresi-Kanals. Generaldirektor Giacinto CAvAGNA di Gualdana erklärte mir seine Pläne und gab mir Schriften und Photographien. Reich mit Eindrücken und Material versehen kehrte ich nach Zürich zurück. Den sehr hilfreichen zuständigen italienischen Herren und Stellen sei herzlich gedankt.

\section{ABGRENZUNG UND BEDEUTUNG DES GEBIETES}

Die Untersuchung beschränkte sich auf die Länder Lombardei und Piemont, und insbesondere auf das Gebiet der Poebene nördlich des Po zwischen Dora Baltea und Adda, das eine Breite von 100 und eine Länge von $200 \mathrm{~km}$ aufweist. In diesem Gebiet befinden sich die größten und wichtigsten Bewässerungsanlagen und -organisationen, sowie die bedeutendste Reisregion der Poebene und Italiens. In ganz Italien sind von $215000 \mathrm{~km}^{2}$ landwirtschaftlich benutztem Areal $14000 \mathrm{~km}^{2}$ oder rund $6 \%$ bewässert. In der Lombardei und im Piemont ist rund ein Drittel des landwirtschaftlich benutzten Areals, das in der Ebene liegende, bewässert. Über Größe und Verteilung des bewässerten Landes in Italien geben folgende Zahlen Auskunft (Irr. in Italia 1931):

\begin{tabular}{|c|c|c|c|c|c|c|c|c|c|c|c|}
\hline & & & 1000 ha bewässert & $\%$ & & & & 1000 & ha & bewässert & $\%$ \\
\hline Italien . & & . & . 1442 & 100 & Piemont & • & $\cdot$ & . & & 480 & \\
\hline Poebene & & . & . 1178 & 81,5 & Lombardei & und & Piem & nont & & 962 & 0 \\
\hline Nördlich des & Po & . & . 1100 & 76,0 & Venezien & .. & . . & . & . & 140 & 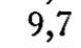 \\
\hline Lombardei & . . & . & 482 & 33,3 & Emilia & . & . & . & . & 77 & \\
\hline
\end{tabular}

\section{NATURVERHÄLTN I!̣S S E}

Die Naturbeschaffenheit (Boden, Klima, Verlauf der Flüsse usw.) setze ich als bekannt voraus. Über die jährliche mittlere Wasserführung der Flüsse und die zur Verfügung stehende Wassermenge informieren folgende Ziffern (Irr. in Italia 1931):

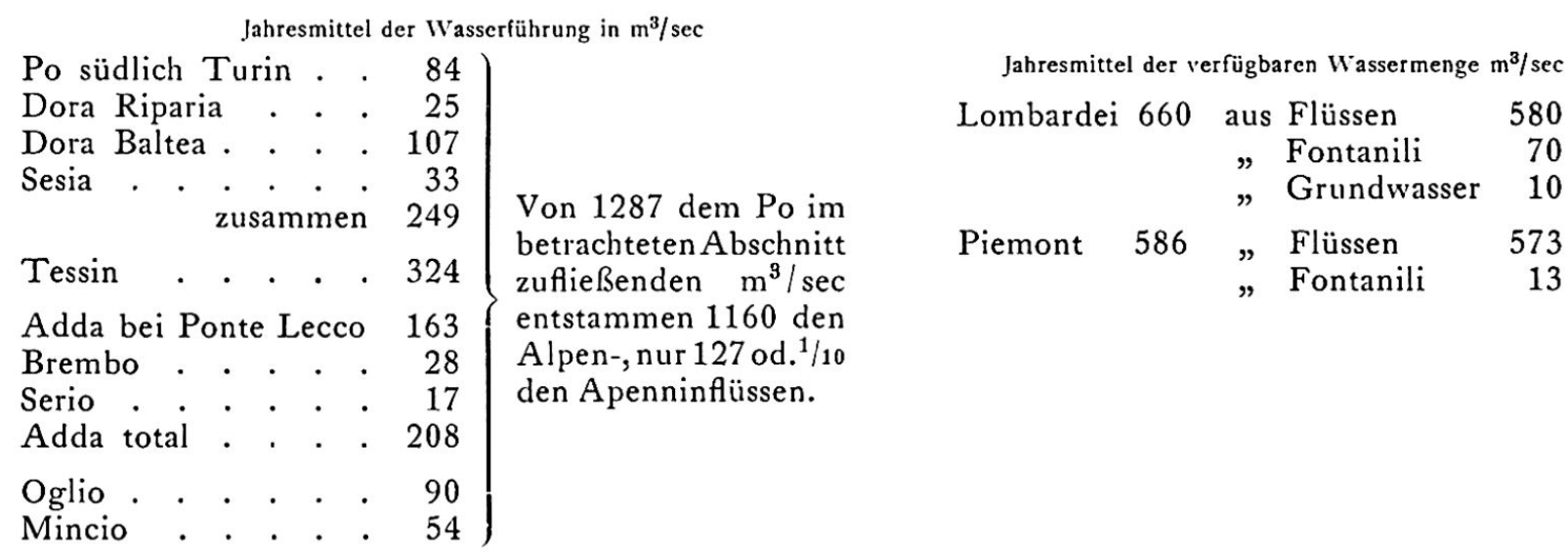

\section{DAS HEUTE BESTEHENDE KANALNETZ}

Die Ebene nördlich des Po zwischen Dora Baltea und Adda weist ein dichtes und vielgestaltiges Kanalnetz auf, das im Verlaufe von tausend Jahren entstanden ist und 
immer noch ausgebaut, verbessert und erweitert wird. Die früheste Anlage von Kanälen geht bis in die erste Hälfte des Mittelalters zurück. Religiöse Genossenschaften (Klöster usw.) sollen dabei eine Rolle gespielt haben. Das Kanalnetz wurde auf handwerklicher Grundlage aufgebaut und betrieben, wobei sich das 12.-15. und 18.-20. Jahrhundert durch rege Bautätigkeit auszeichnen. Die großen Bauten des Cavour- und Villoresikanals, diese größten Bewässerungswerke Italiens, entstanden in der zweiten Hälfte des vergangenen Jahrhunderts nach der italienischen Einigung. Der Regina Elenakanal, die Zuleitung zum Cavourkanal aus dem Tessin, wurde am 1. Juli 1954 eröffnet. Sein Bau erfolgte im Anschluß an die 1942 beendete Regulierung des Langensees. Die Kanalgeschichte wäre zweifellos kulturgeschichtlich höchst reizvoll und interessant.

Der von Chivasso am Po nö Turin bis nö Novara zum Tessin führende Cavourkanal ist $82 \mathrm{~km}$, das gesamte Netz der Cavourkanäle, d.h. der piemontesischen Staatskanäle (Canali Demaniali d'Irrigazione - Canali Cavour) aber über $2200 \mathrm{~km}$ lang. Es befördert eine Wassermenge von $350 \mathrm{~m}^{3} / \mathrm{sec}$. und bewässert über 200000 ha. Und das ist nur ein Teil der piemontesischen Kanäle. Die Associazione Irrigazione Est Sesia verwaltet in ihrem Gebiet zwischen Sesia und Tessin, dessen südlicher Teil, die Lomellina, zur Lombardei gehört, gegen $2000 \mathrm{~km}$ Kanäle. Der vom Tessin über Monza zur Adda führende Villoresikanal ist $86 \mathrm{~km}$ lang; seine Sekundärkanäle sind $130 \mathrm{~km}$ und die Tertiärkanäle $1400 \mathrm{~km}$ lang, macht zusammen $1616 \mathrm{~km}$. Die Carte delle Irrigazioni Lombarde und Piemontesi von 1929/30 enthalten ausführliche Kanalverzeichnisse. Sie nennen: von Flüssen abgeleitete Hauptkanäle (ohne die zahlreichen Abzweigungen): Lombardei 97, Piemont 137. Von Fontanili abgeleitete Hauptkanäle (ohne Nebenkanäle) : Lombardei 223, Piemont 22. Macht total Hauptkanäle: Lombardei 320, Piemont 159. Die Wasserführung der Fontanilikanäle ist in der Regel geringer als jene der Flußkanäle.

Angesichts des großen Umfangs des lombardischen und piemontesischen Kanalnetzes kann mit Recht von einer Verlegenheit oder Verwirrung aus Überfluß gesprochen werden. Jeder einzelne Hauptkanal bildet mit seinen Verzweigungen eine Einheit für sich und müßte besonders beschrieben werden. Daher ist es auf dem hier gegebenen Raume nur möglich und auch genügend, die größten und wichtigsten Kanäle und Kanalsysteme (d. h. jene mit mehr als $10 \mathrm{~m}^{3} / \mathrm{sec}$. oder mehr als 10000 ha Bewässerungsfläche) zu nennen und kurz zu charakterisieren, um einen Überblick über die Grundstruktur dieser Kanallandschaft zu gewinnen.

Die großen Kanäle des Piemont zwischen Dora Baltea und Tessin (Canali Demaniali Cavour u. a. m.)

\begin{tabular}{lrc}
\multicolumn{1}{c}{ Name } & Länge km & Eröfnung \\
1. Cavourkanal (Po-Tessin) & 82 & 1866 \\
Zuleitungen zum Cavourkanal & & \\
2. Farinikanal (Dora Baltea) & 3 & 1868 \\
3. Regina Elenakanal (Tessin) & 25 & 1954 \\
Ableitungen des Cavourkanals (östlich der Sesia) & \\
4. Cavo Montebello & 19 & 1868 \\
5. Diramatore Quintino Sella & 24 & 1871 \\
a) Subdiramatore destra Mortara & 15 & \\
b) Subdiramatore sinistra Pavia & 35 & \\
6. Diramatore Vigevano & 18 & 1868 \\
Kanäle westlich der Sesia & & \\
7. Naviglio d'Ivrea & 70 & 1468 \\
8. Depretiskanal & 40 & 1785 \\
9. Del Rottokanal & 13 & 1400 \\
östlich der Sesia & & \\
10. Roggia Mora & 50 & 1481 \\
11. Roggia Busca & 55 & 1380
\end{tabular}




$\begin{array}{lcr}\text { 12. Roggia Biraga } & 52 & 1488 \\ \text { 13. Roggione di Sartirana } & 17 & 1387 \\ \text { 14. Naviglio Langosco } & 30 & 14 . \mathrm{Jh} \text {. } \\ \text { 15. Naviglio Sforcesco } & 40 & 1402 \\ \quad \text { Hebewerke an der Dora Baltea } & & \\ \text { 16. Elevatore idraulico di Cigliano } & 1700 \mathrm{1} / \mathrm{sec} / 40 \mathrm{~m} \mathrm{hoch} & 1889 \\ \text { 17. Impianto elevatore di Villaregia } & 5000 \mathrm{1} / \mathrm{sec} / 60 \mathrm{~m} \text { hoch } & 1927 \\ \text { 18. Inpianto elevatore di Mazzè } & 2270 \mathrm{1} / \mathrm{sec} / 30 \mathrm{~m} \text { hoch } & 1928\end{array}$

Fast alle piemontesischen Kanäle sind miteinander zu einem vielfältig kummunizierenden System verbunden, das besonders der Bewässerung der Reisgebiete von Vercelli, Novara und Mortara (Lomellina) dient. Hauptarterie ist der Cavourkanal. Erwähnenswert sind ferner die Kraft- und Hebewerke an der Dora Baltea bei Cigliano, Villaregia und Mazzè, durch die das Flußwasser auf die dort höher gelegene Ebene gepumpt wird.

Die großen Kanäle in der Lombardei zwischen Tessin und Adda (Region von Mailand) :

$\begin{array}{lcr}\text { 19. Villoresikanal (Tessin - Adda) } & 86 & 1892 \\ \text { 20. Industriekanal von Vizzola } & 20 & 20 . \text { Jh. } \\ \text { 21. Naviglio Grande } & 40 & 1179 \\ \text { 22. Naviglio di Bereguardo } & 30 & 15 . \text { Jh. } \\ \text { 23. Naviglio della Martesana } & 35 & 1460 \\ \text { 24. Naviglio di Pavia } & 30 & 14 \text {. Jh. } \\ \text { 25. Muzzakanal } & 70 & 1220\end{array}$

In der Lombardei befinden sich zwischen Tessin und Adda sieben große Kanäle, wovon die Kanäle 20-24 ein zusammenhängendes System bilden. In den Industriekanal von Vizzola sind sechs Kraftwerke eingebaut. Auf den Naviglii können Lastkähne verkehren zwischen Mailand, 'Tessin, Adda und Po. Ein Naviglio darf keine Dücker oder Unterführungen (Sifone) aufweisen, und die Strömung darf nicht zu schnell sein. Der Villoresikanal wird von einer Genossenschaft betrieben. Alle andern sind Staatskanäle.

Staung und Regulierung der Alpenseen. Zum Zweck der Verbesserung der Bewässerung und Kraftnutzung sind in den letzten Jahrzehnten die Alpenseen durch Einbau von Regulierwehren in den Auslauf in künstliche Speicherbecken verwandelt worden. Und zwar betrifft dies im Bereiçhe des Piemont und der Lombardei: Orta-, Langen- (seit 1942), Vareser-, Comer- (1944), Iseo- (1933), Idro- und Gardasee. Art und Wirkung dieser Stauung sei nur am Beispiel des Langensees kurz beschrieben. Das Langenseebecken umfaßt mit allen Zuflüssen eine Fläche von $6600 \mathrm{~km}^{2}$, wovon 3370 in der Schweiz und 3230 in Italien liegen. Der Langensee ist $210 \mathrm{~km}^{2}$ groß. Ein Zentimeter Niveauunterschied enthält demgemäß $2100000 \mathrm{~m}^{3}$. Die bewilligte Regulierhöhe beträgt $2 \mathrm{~m}$, was einer Wassermenge von 420 Millionen $\mathrm{m}^{3}$ entspricht. Die Nutzung fällt in die Monate der sommerlichen und winterlichen Trockenheit. Die Seen dienen zugleich zur so wichtigen Vorwärmung des Wassers. Der Tessin, dessen Wasser zwischen Piemont und Lombardei geteilt wird, speist bald nach seinem Ausfluß aus dem Langensee neun Kanäle: Auf dem rechten Ufer: 1. Regina Elenakanal, 2. Roggia di Oleggio, 3. Naviglio Langosco, 4. Naviglio Sforzesco, 5. Roggia di Galliate, 6. Roggia Castellana und Magna. Auf dem linken Ufer: 7. Villoresikanal, 8. Vizzolakanal, 9. Naviglio Grande.

Anlage und Verlauf der Kanäle. Die ältesten Kanäle, die noch nicht besonders lang und tragfähig waren, zweigten an geeigneten Stellen von den Flüssen Wasser ab und leiteten es, meist mehr oder weniger parallel zum Fluß dem natürlichen Gefälle folgend, in das umliegende Land. Kanäle, die derart neben dem Fluß herlaufen und oft wieder in ihn zurückkehren, sind z. B. die Roggie Busca und Biraga, die Roggia di Oleggio, die Naviglii Langosco, Sforzesco und Bereguardo und der Muzzakanal. Die größten und wichtigsten Kanäle aber zeigen einen andern Verlauf. Dora Baltea, Tessin und Adda durchbrechen die z. T. mächtigen Moränen und Schotter am Alpensüdrand in canonartigen Einschnitten. Diese Engen, in denen die Flüsse fixiert sind, 


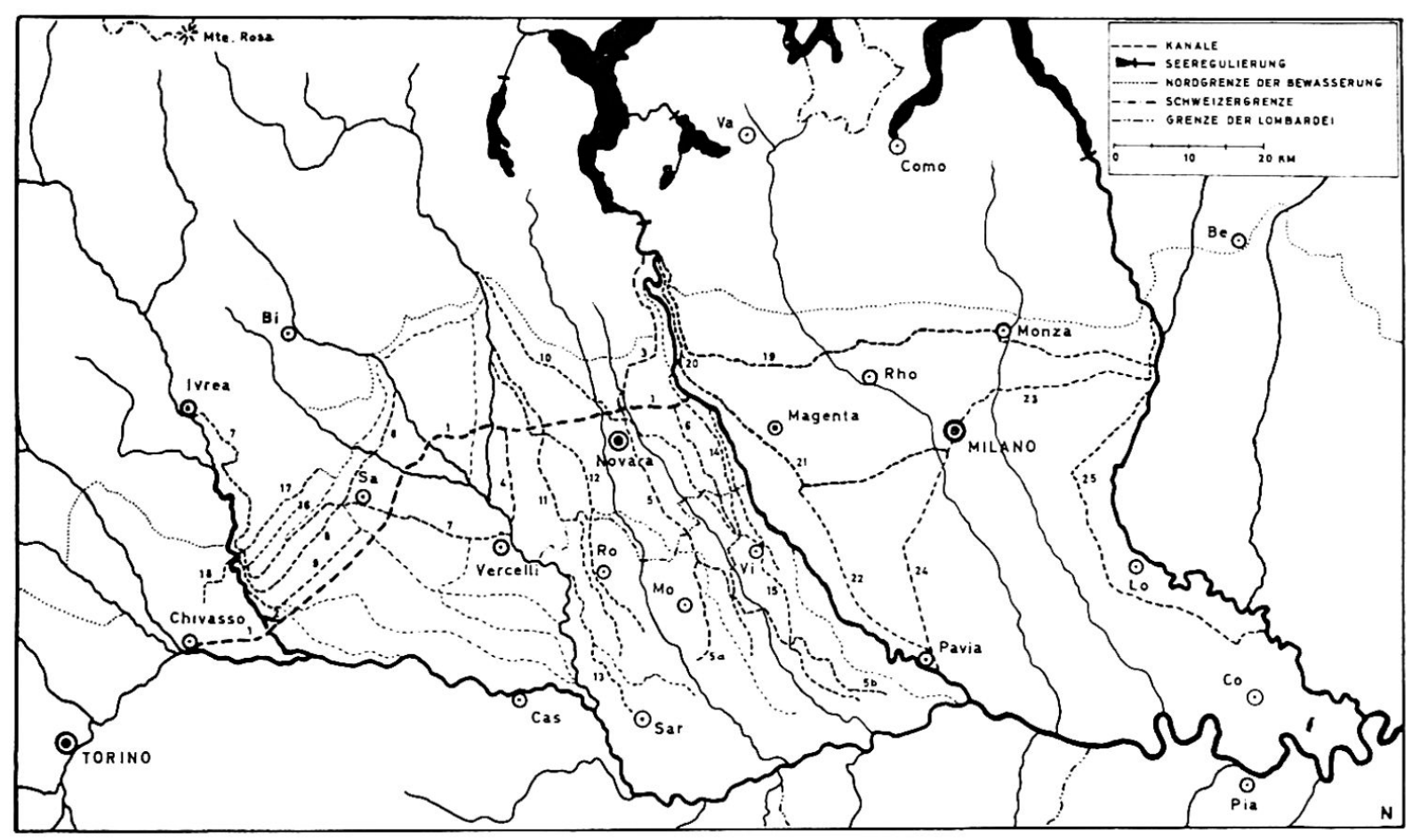

Bewässerungsnetz zwischen Dora Baltea und Adda.

Die Nummern der Kartenskizze beziehen sich auf die im Textverzeichnis genannten Kanäle.

eignen sich besonders gut für Stauung und Wasserentnahme. In dieser Zone befinden sich die wichtigsten Kanalfassungen. Die Kanäle laufen in den Talengen zunächst dem Fluß parallel, um dann außerhalb des Moränengürtels umgeleitet und parallel zum Alpenrand und quer zu den Flüssen über die Ebene geführt zu werden. Aus diesen Hauptkanälen wird das Wasser dann in geeigneten Abständen und an geeigneten Stellen zu seinem $Z$ wecke über die leichtgeneigte Ebene powärts abgelassen und verteilt. Cavour- und Villoresikanal sind auf diese Weise angelegt; aber auch die folgenden älteren Kanäle: Naviglio d'Ivrea und Depretiskanal, Roggia Mora, Naviglio Grande und Naviglio della Martesana.

Der bekannte Gegensatz zwischen den groben und durchläßigen Schotterböden längs des Moränengürtels am Alpenrand und den feinen Schwemmlandböden am Unterlauf der Flüsse ist nicht ohne Einfluß auf Anlage und Verlauf der Kanäle geblieben. Diese befinden sich überwiegend im feinen lehmigen Schwemmland; denn auf den groben Schotterböden ist der Wasserverlust durch Versickerung zu groß. Wo sie trotzdem über Schotter geführt werden müssen, muß ihr Bett ausgemauert werden, was die Anlagekosten vergrößert. An der Grenze zwischen Schotterböden und feinem Schwemmland befinden sich ja auch die Fontanili. Auch die Reiskultur ist an das feine Schwemmland gebunden. Auf diesen natürlichen Verhältnissen beruht der bekannte Gegensatz zwischen trockener und feuchter Poebene.

Cavourkanal und Cavourkanäle. Seit 1862 ist dies die Bezeichnung der ehemaligen piemontesischen Staatskanäle, die seit 1400 vom Hause Savoyen systematisch angelegt, erweitert und ausgebaut wurden «zum Nutzen der Wirtschaft und des Staates, zun Vorteil der Landwirtschaft, der Industrie und des Verkehrs». Der älteste ist der um 1400 eröffnete Del Rottokanal. Ihm folgten 1468 der Naviglio d'Ivrea, der auch dem Salztransport diente, 1785 der Depretiskanal. Dieses Staatsnetz wurde durch Neubauten und besonders durch Ankauf bereits bestehender privater Kanäle vergrößert. Zur Zeit der Einigung Italiens um 1861, also noch vor dem Bau des Cavourkanals, umfaßte es bereits $1200 \mathrm{~km}$ mit einer Leistung von $120 \mathrm{~m}^{3} / \mathrm{sec}$. Das Wasser wurde hauptsächlich aus der Dora Baltea genommen; denn solange der Tessin die Grenze «Ita- 
liens» gegen Österreich bildete, kam seine Nutzung für Piemont nicht in Frage. Bis 1800 waren die Staatskanäle der Wirtschaftsabteilung des Innenministeriums unterstellt. Seither gehören sie zum Finanzministerium. Nach der Einigung Italiens wurde die Verwaltung vom italienischen Staat, also von der Regierung in Rom, übernommen. 1863-66 wurde der große Cavourkanal gebaut. 1954 wurde endlich durch die Inbetriebnahme des Regina Elenakanals die sehr willkommene und längst gewünschte Ergänzung der Wassermenge der Cavourkanäle östlich der Sesia aus dem Tessin verwirklicht.

Das System der Cavourkanäle ist die größte und wichtigste Bewässerungsanlage Italiens. Es umfaßt $2200 \mathrm{~km}$ Kanäle mit einer Leistung von $350 \mathrm{~m}^{3} / \mathrm{sec}$. Zahlreiche Kraftwerke und Mühlen sind darin eingebaut. Es verteilt sich über die sechs Provinzen Turin, Aosta, Vercelli, Alessandria, Novara und Pavia. Die dem Finanzministerium in Rom unterstellte Amministrazione generale dei canali demaniali d'irrigazione (Canali Cavour) befindet sich in Turin. Die Verwaltung erfolgt über acht regionale Zentren in Chivasso, Santhià, Vercelli, Novara, Mortara, Sartirana, Robbio-Lomellina und Casale Monferrato; sowie durch die zwei großen Bewässerungsgenossenschaften westlich und östlich der Sesia. «Ebenso alt wie die Technik des piemontesischen Bewässerungswesens ist dessen Organisation in Genossenschaften». (H. BERNHARD).

\section{DIE ORGANISATIONEN}

Associazione d'Irrigazione dell'Agro all'Ovest del Sesia in Vercelli. Diese Vereinigung wurde 1853 im Hinblick auf den geplanten Bau des Cavourkanals gegründet. In ihr wurden die Bewässerungsinteressenten zwischen Dora Baltea und Sesia zusammengefaßt. Nach der Gründung gehörten ihr 3500 Landeigentümer mit 15000 ha an. Sie war damals eine der größten, wenn nicht die größte landwirtschaftliche Genossenschaft in Europa und richtungsweisend für die Bewässerungskultur Italiens. Heute umfaßt diese Vereinigung 25000 Landeigentümer mit 60000 ha. Sie besitzt und bewirtschaftet über $2000 \mathrm{~km}$ eigene Kanäle, die an das Netz der Staatskanäle angeschlossen sind. Ihr Organisationsstatut ist berühmt. Es ist die Aufgabe der Vereinigung, in ihrem Gebiet den Verkehr mit der Staatsverwaltung zu besorgen, das Wasser unter den Besitzern zu verteilen, die Kanäle zu unterhalten, auszubauen und zu verbessern, neues Land der Bewässerung zu erschließen usw. Die Zentralverwaltung befindet sich in Vercelli. Einige Einzelheiten über den Betrieb einer solchen Bewässerungsgenossenschaft werden im nächsten Abschnitt mitgeteilt.

Associazione Irrigazione Est Sesia in Novara. Diese Gesellschaft ist heute die größte Bewässerungsgenossenschaft Italiens. Sie verwaltet und bearbeitet Ländereien im Umfang von 200000 ha mit einem Kanalnetz von rund $2000 \mathrm{~km}$ Länge. Infolge der besonderen politischen Grenzverhältnisse war die Bewässerung zwischen Sesia und Tessin vernachläßigt. Sie beschränkte sich auf partikuläre Bewässerungsanlagen religiöser Genossenschaften, großer Feudalherren und freier Gemeinden. Die piemontesischen Staatskanäle reichten nicht ostwärts der Sesia. Der Bau des Cavourkanals bis zum Tessin war u. a. ausdrücklich dazu bestimmt, die Region östlich der Sesia an das System der piemontesischen Staatskanäle anzuschließen und ihr durch den Cavourkanal eine Wassermenge von $90 \mathrm{~m}^{3} / \mathrm{sec}$ zuzuführen. Infolge von eintretenden Schwierigkeiten blieb die Bewässerung dieses Gebietes aber auch weiterhin gehemmt. Sie funktioniert erst seit Eröffnung des Regina Elenakanals (1954) und seiner zusätzlichen Wasserzufuhr aus dem Tessin befriedigend.

Nach jahrzehntelangen vergeblichen Bemühungen wurde 1923 die bestehende Vereinigung zuerst probeweise und 1929 endgültig gegründet. Sie umfaßt die Wasserverbraucher zwischen Sesia, Po und Tessin. Sie bezweckt die rationelle Bewirtschaftung und Verbesserung der Wasserverteilung. In einer der Schriften der Gesellschaft heißt es: «Die durch sie geleistete Arbeit ist von größtem Ausmaß. Handelte es sich 


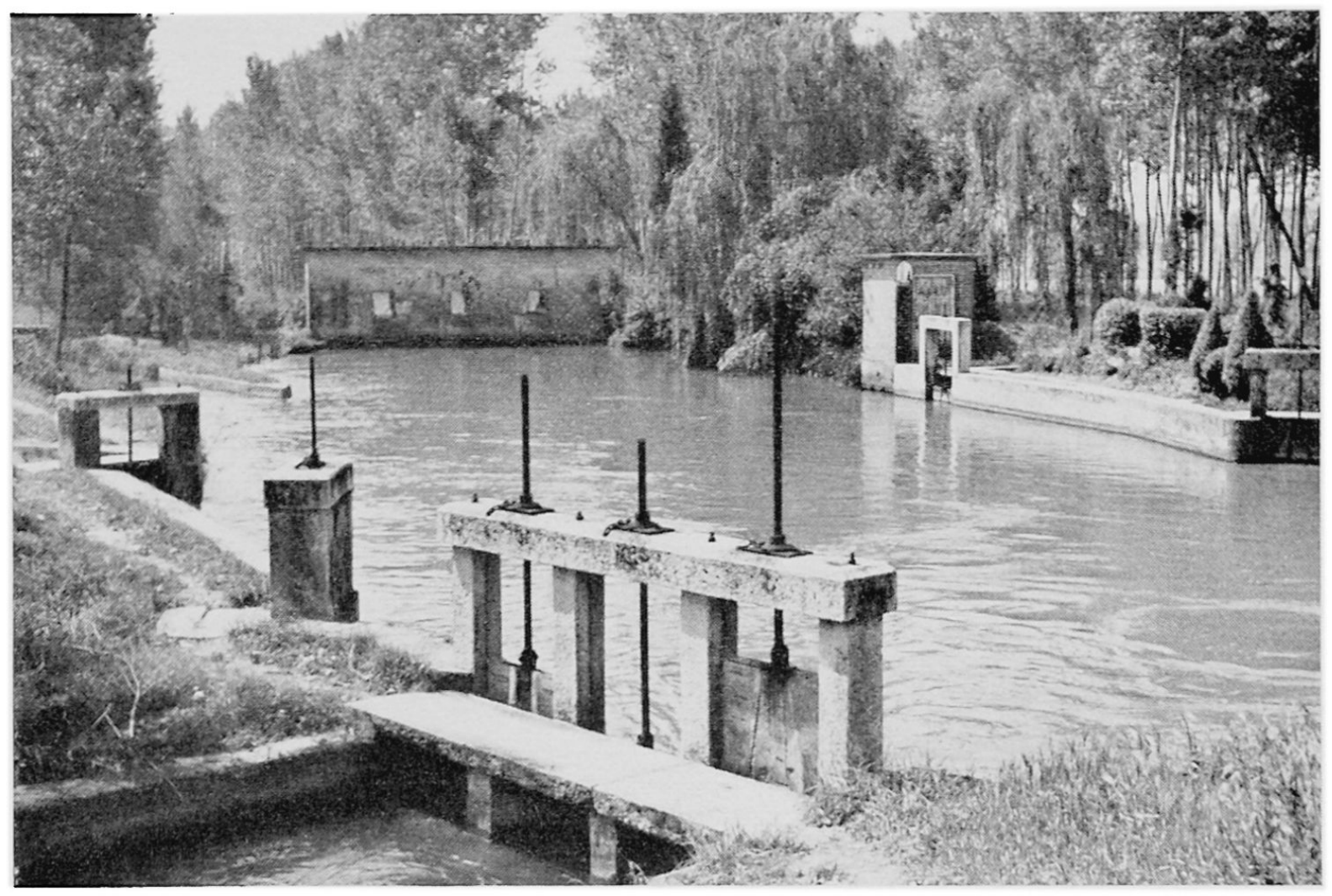

Wasserverteilung aus einem großen in kleine Kanäle zwischen Vercelli und Novara (Photo W. NELz)

doch darum, eine mehr oder weniger chaotische, aus dem Mittelalter stammende Bewässerung mit Anlagen früherer Zeiten, die doch stets unter Berücksichtigung individueller Interessen errichtet worden waren, durch eine rationelle und geordnete Verwaltung zu verbessern und auszubauen, was entsprechend den Erfordernissen des gesamten verwalteten Gebiets durchgeführt wurde». Kanäle wurden umgebaut, aufgehoben, zusammengefaßt und neu angelegt, um neue Flächen der Bewässerung zu erschließen. Was diese Arbeit bedeutet, kann man ermessen, wenn man weiß, wie gerade in Bewässerungsfragen die grundlegenden Lebensinteressen oft hart aufeinanderstoßen. Durch Ausmauerung der Kanäle auf durchläßigen Böden wurden die Versickerungsverluste von 30 auf $10 \%$ vermindert.

Die Zentralverwaltung der Gesellschaft hat vor einiger Zeit mitten im alten Novara einen stattlichen Neubau bezogen. Sie wird geleitet von einer Generaldirektion, einem Verwaltungsrat und einer Delegiertenversammlung. Das Gebiet ist in zwölf Zonen mit eigenen Verwaltungen eingeteilt. Das Bewässerungsnetz der Gesellschaft zählt rund 200 unter einander verbundene große und kleine Kanäle. Über das ganze Gebiet sind etwa 80 Wärterhäuser verteilt, die mit der Zentrale in Novara in Verbindung stehen. Der Wasserstand in den Kanälen wird täglich zweimal, morgens und abends, registriert, an die Zentrale gemeldet und von ihr aus reguliert. Die Gesellschaft hat sich seit ihrer Gründung rasch und stetig entwickelt. 1929 betrug das Netz ihrer Hauptkanäle $656 \mathrm{~km}$. Es waren die von ihr in Verwaltung übernommenen Staatskanäle. 1954 umfaßte es $1670 \mathrm{~km}$, einschließlich Elenakanal. Mehr als die Hälfte dieses Netzes sind von Privaten und Gemeinden in Verwaltung übernommene Kanäle. Heute ist es rund $2000 \mathrm{~km}$ lang. 1929 betrug die zur Verfügung stehende Wassermenge $140 \mathrm{~m}^{3} / \mathrm{sec}, 195+215 \mathrm{~m}^{3} / \mathrm{sec}$. Dieses Wasser kommt fast zur Hälfte aus Po und Dora Baltea durch den Cavourkanal. 25\% stammen aus dem Tessin durch den Elenakanal, 20\% aus der Sesia, und der Rest von ca. 7\% fließt aus Quellen (Fontanili) und lileinen Flüssen. 1929 umfaßte das Reisland 45000 ha, 195365000 ha. Auf 
die wichtigen und komplizierten Fragen der Kosten und der Wasserpreise (die Benützer zahlen Wasserzins), sowie die Art und Weise der Wasserentnahme (die Kehrordnung) kann hier nicht eingegangen werden, weil das zu weit führen würde.

Consorzio Villoresi in Mailand. Das Consorzio Villoresi ist ähnlich organisiert wie die erwähnten Genossenschaften. Der Villoresikanal (seit 1892 in Betrieb) ist aber nicht Staatskanal, sondern er gehört der Vereinigung. Die Villoresi-Gesellschaft wurde 1912 gegründet und übernahm den Kanal 1918 von der Società Italiana per Condotte d'Acqua, die ihn gebaut hat. Der Kanal besitzt auf seiner Länge von $86 \mathrm{~km} 120 \mathrm{Ab}$ zweigungen. Sein gesamtes Netz hat eine Länge von über $1600 \mathrm{~km}$. Er bewässert westlich, nördlich und nordöstlich von Mailand eine Zone von 58000 ha. Diese Zone, die gegen Süden bis zum Naviglio Grande und Naviglio della Martesana reicht, ist westlich von Mailand $15 \mathrm{~km}$, an der Adda nur noch $2 \mathrm{~km}$ breit. Die Bevölkerungsdichte dieses stark industrialisierten Gebiets beträgt ohne Mailand und Monza über $600 \mathrm{Ew} . / \mathrm{km}^{2}$. Nur $10 \%$ der Bevölkerung dieser Zone sind hauptberuflich in der Landwirtschaft tätig; weitere $10 \%$ nebenberuflich. Der Boden ist sehr stark zersplittert; überwiegend bestehen Klein- und $Z$ wergbetriebe, die von ihren Eigentümern bewirtschaftet werden. Die mittlere Betriebsgröße beträgt $670 \mathrm{~m}^{2}$. Die Bodenbenützung zeigt folgende Gliederung: Kunstwiesen 23\%, Mais 30\%, Weizen 30\%, Roggen und Hafer 6\%, Reis 1\%, Anderes 10\%.

Der Villoresikanal wurde errichtet, um den Niedergang der Seidenraupenzucht durch Bodenverbesserung auszugleichen. Die Besonderheit dieses Kanals besteht darin, daß er - nördlich von Mailand - über Schotterböden, d. h. über die trockene Poebene führt. Sein Hauptproblem ist deshalb der Wasserverlust durch Versickerung in den Kanälen. Als die Villoresi-Gesellschaft den Kanal übernahm, versickerten von $70 \mathrm{~m}^{3} / \mathrm{sec} 42$ oder $60 \%$ in den Kanälen. Die Gesellschaft konnte seither den Wasserverlust durch systematische Abdichtung der Kanäle auf $28 \mathrm{~m}^{3} / \mathrm{sec}$ oder rund $1 / 3$ senken. Infolge der Regulierung des Langensees und Verringerung des Versickerungsverlusts ist es durch vermehrte Wassermenge möglich geworden, im Gebiete nördlich des Kanals weitere 27000 ha durch Bewässerung zu verbessern, sodaß die gesamte bewäßerte Fläche gegenwärtig 85000 ha beträgt. In den letzten Jahren hat man begonnen, neben der Kanalbewässerung auch die Regenbewässerung durch Röhren anzuwenden, was Wasser spart. Die Regenbewässerung bewährt sich vor allem in hügeligem Gelände.

Die Generaldirektion der Villoresigesellschaft befindet sich in Mailand. Sie unterhält Regionalverwaltungen in Magenta, Rho und Monza, sowie ein Versuchsfeld in Marcallo. Der Generaldirektor plant, die Gesellschaft zu erweitern und nach dem Vorbild der piemontesischen Bewässerungsgenossenschaften das ganze lombardische Gebiet zwischen 'Tessin, Po und Adda ihrer Verwaltung zu unterstellen. Doch scheint sich dieser Plan nicht leicht realisieren zu lassen.

\section{BEWÄSSERUNGSANLAGEN, STAAT UND GEISTESKULTUR}

Wie schon einleitend erwähnt, können große Wasserbauten nur durch organisiertes Zusammenwirken der gesamten im Bereiche eines Gewässers lebenden Bevölkerung ausgeführt werden. Das Leben spendende und vernichtende Wasser wird zur Sache des allgemeinen Interesses oder Willens, der öffentlichen Macht, d. h. des Staates. Auch am Po sind die großen Wasserbauten von Anfang an durch Kooperation Vieler errichtet und in Stand gehalten worden. Zunächst befaßten sich religiöse Genossenschaften und Verbände (Klöster und Bistümer), Gemeinden und Feudalherren, später die Staatsmacht, mit ihnen. Die gesamte Wasserwirtschaft wurde gesetzlich geregelt. Der Staat ist direkt durch seine Organe der oberste Organisator. Die Staatskanäle bilden das Rückgrat der Bewässerungsanlagen. Die einzelnen Wasserbenützer sind zur Durchführung ihrer besondern Aufgaben in großen, mit dem Staat kooperie- 


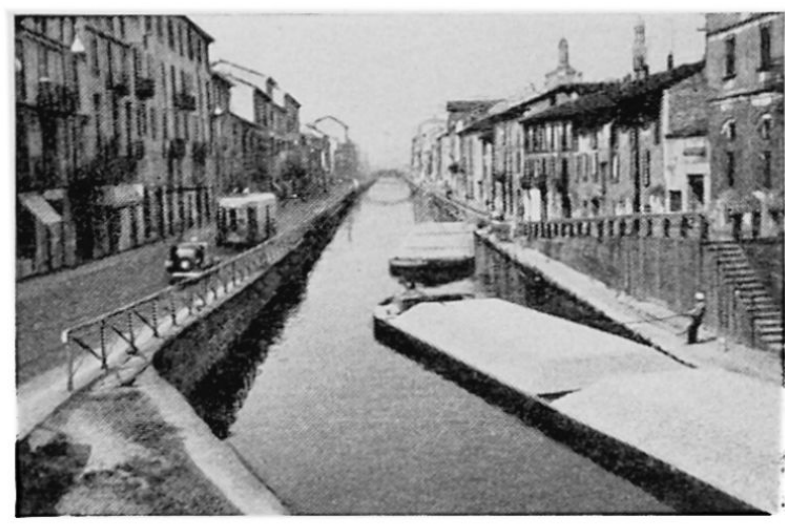

Naviglio Grande in Mailand vor der Einmündung in den Hafen

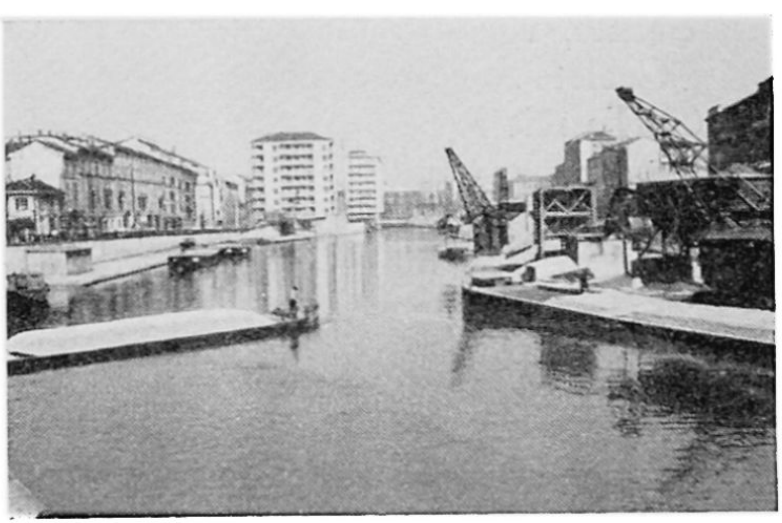

Hafen von Mailand (Photos W. NeLz)

renden Verbänden zusammengefaßt. Auch die Bewirtschaftung der Flüsse ist besondern Gesellschaften anvertraut; den Konsorzien des Tessins, der Adda, des Oglio usw. Auf diese Weise erhält die sozial verbindende Funktion des Wassers eine viel ausgedehntere, tiefere, intensivere Gestaltung als durch die bloß verkehrsmäßige Verbindung, an die gewöhnlich gedacht wird, und die am Po außerdem stark zurücktritt. Landwirtschaft, Industrie und Verkehr sind durch das Wasser einerseits mit der Natur und andrerseits mit dem allgemeinen gesellschaftlichen Prinzip und Organ zu konkreter 'Totalität verbunden. Die Fäden dieses komplizierten, über das ganze Land ausgebreiteten Systems, laufen wie die Drähte des betriebseigenen Telephons in den städtischen Zentralen zusammen. Auch das Geistesleben steht begreiflicherweise unter dem Einfluß der Wasserwirtschaft. Die Tatsache, daß die ältesten Bewässerungskanäle im Piemont und der Lombardei von religiösen Genossenschaften ausgingen, ist ein Hinweis auf religiöse Zusammenhänge. Ein Werk, das jahrhundertelang ununterbrochen große Teile der Bevölkerung beschäftigt, wird sicher auch in der Sprache, durch die sich die Menschen verständigen, hör- und sichtbare Zeichen und Zeugnisse hinterlassen. Ein Blick auf die topographische Karte Oberitaliens genügt, um eine Reihe von Spezialausdrücken herauslesen zu können, die für die Kanäle zur Bezeichnung ihrer technischen und regionalen Verschiedenheiten in Gebrauch gekommen sind. Solche Ausdrücke sind außer den allgemeinen Canale und Acquedotto, Naviglio und Navilotto für schiffbare Kanäle, Roggia, Roggione, Seriola, Serioletta, Bealera, Bedale, Dugale, Gora, Vaso, Fossa und Fosso, Cavo, Diramatore, Subdiramatore für Bewässerungskanäle und -gräben; Scolo, Colatore, Raccogliatore für Wassersammler und Abflußgräben; Fontane, Fontanone, Fontanili, Fontanini, Sillaro, Colo, Bocca, Bocchello, Bocchetto, Sorgente, Sorgino für Quellen und Brunnen usw. Ein wasserbauendes Volk hat also in seinem Sprachschatz Ausdrücke und Wendungen, die mit dem Wasserbau zusammenhängen, die in der Sprache eines Volkes, das den Wasserbau nicht kennt, gar nicht entstehen können. Auch das in Oberitalien bekannte Wort «Der Reis entsteht im Wasser und stirbt im Wein» deutet in diese Richtung.

\section{DER BEWÄSSERUNGSLANDBAU}

Ausgesprochene Bewässerungskulturen sind der Reisbau und die Wasser- oder Rieselwiesen, die Marcite. Das Jahr ist infolge des Klimas in die Bewässerungsperioden des Sommers und des Winters eingeteilt. Die Zeit der Entleerung der Kanäle im Herbst wird für Reinigung und Reparaturen verwendet. Für die verschiedenen Kulturen wird während der Bewässerungszeit folgender Wasserverbauch in Sekundenlitern pro ha angegeben: Wiesen $0,8-2$; Reis $2-5$; übrige Äcker (Mais, Weizen usw.) 0,2-1,2; Marcite 30-50. Die Wässerwiesen brauchen am meisten Wasser.

Die Reiskultur: Die westliche Poebene ist für uns der nächstgelegene Ort, wo die 
interessante, von dem uns gewohnten Ackerbau stark unterschiedene Reiskultur, die weite Gebiete Asiens bestimmend beeinflußt, zu beobachten ist. 1952 wurden in Italien 174000 ha mit Reis angepflanzt, 99\% davon in der Poebene, 91\% im Piemont (50) und der Lombardei (41). Hier konzentriert sich der Reisbau auf die Provinzen Vercelli (60 000), Pavia (50 000), Novara (23 000) und Mailand (18000 ha). Der italienische Reisbau geht bis auf das 15. Jahrhundert zurück. Bis ins 19. Jahrhundert war die Lombardei führend (Mailänder Reis) infolge ihrer damals bessern Bewässerungsanlagen. Seither ging der Reisbau in der Lombardei, angeblich infolge asiatischer Konkurrenz seit Eröffnung des Suezkanals, stark zurück, während er sich im Piemont im Anschluß an den Bau des Cavourkanals rasch und stetig bis in die Gegenwart ausbreitete. Von 1931 - 1953 stieg die Reisanbaufläche in Italien von 130 auf 182000 ha.

Die Besonderheit der Reisbewässerung besteht darin, daß das Wasser langsam durch die Reisfelder fließen, d. h. sowohl zugeführt als auch abgeleitet werden muß. Alle Feldarbeiten bis zur Ernte müssen unter Wasser ausgeführt werden. Die Versetzung der jungen Reispflanzen aus den Saatbeeten auf die Felder geschieht im Juni. Nachher muß der Wasserstand den wachsenden Reispflanzen angepaßt werden. Die Reisernte ist Ende September-Oktober. Zum Studium und zur Erprobung des Reisbaus und überhaupt der Bewässerungskulturen wurde 1908 in Vercelli die Stazione Sperimentale di Risicoltura, die ein Mustergut unterhält, errichtet.

Auf den ersten Blick hat man den Eindruck, daß der große Bewässerungsanlagen erfordernde Sumpfreisbau viel mehr Arbeitsaufwand benötigt als der uns bekannte Regenfeldbau. Außerdem müssen die wichtigsten Feldarbeiten unter Wasser ausgeführt werden, was wohl auch keine Erleichterung darstellt. Man behauptet, daß es der Hirte, sei er Grasbauer oder Nomade, leichter hat als der Ackerbauer. Aber unter Ackerbauern scheint es der Regenfeldbauer doch wesentlich leichter zu haben als der Reisbauer. Ich vermute, daß dieser Unterschied eine Ursache für die verschiedene Entwicklung Asiens und Europas darstellt. Solange die handwerkliche Landwirtschaft die Haupttätigkeit der Menschheit darstellt, müssen solche Unterschiede ins Gewicht fallen.

Die Wässerwiesen oder Marciten sind Winterwiesen. Das aus Fontanili und Scen bezogene Wasser, das eine Temperatur von $5-10^{\circ}$ aufweist, wird im Winter in erster Linie zur «Heizung» der Wiesen verwendet. Das Wasser muß reichlich durch die Wiesen fließen, um die Bodentemperatur auf der benötigten Höhe zu halten, weshalb verhältnismäßig viel Wasser gebraucht wird. «Die Folge ist, daß das Wachstum der Futterpflanzen sich auch vollzieht, wenn die gewöhnlichen Wiesenflächen öde liegen. Das Gras wächst sozusagen unter dem Schnee ... Die Winterbewässerung dauert etwa 4 $1 \frac{1}{2}$ Monate». (H. Bern haRD). Im Winter fallen die Marcite durch ihr frisches Grün auf. Mit einem ergänzenden Hinweis auf den Bodenbesitz sei geschlossen. Im feuchten Teil der Poebene südlich von Mailand, vor allem aber in den Bewässerungslandschaften des Piemont, d.h. im Reisland, überwiegt der Großgrundbesitz mit Pachtwirtschaft. Am ausgeprägtesten ist er im Novarese. Die Bewässerungswirtschaft begünstigt die Großbetriebe. Bewässertes Land ist wertvoller und teurer als trockenes Land.

\section{A U S B L I C K}

Mir scheint, daß bisher die großen Bewässerungskulturen, einschließlich die lombardische und piemontesische, von der Anthropogeographie trotz relativ zahlreichen Arbeiten hierüber nicht entsprechend ihrer geschichtlichen Bedeutung gewürdigt worden seien. Dabei handelt es sich um einen sehr konkreten und optisch eindrücklichen Gegenstand mit vielseitigen Aspekten, der sich für geographische Darstellungen ausgezeichnet eignet. Auch ist zu beachten, daß die Kanalsysteme der Bewässerungslandschaften ursprünglich den intensivsten realen territorialen Zusammenschluß darstellten, dies übrigens auch heute noch, weil die Bewässerungsanlagen als Bestandteile des 


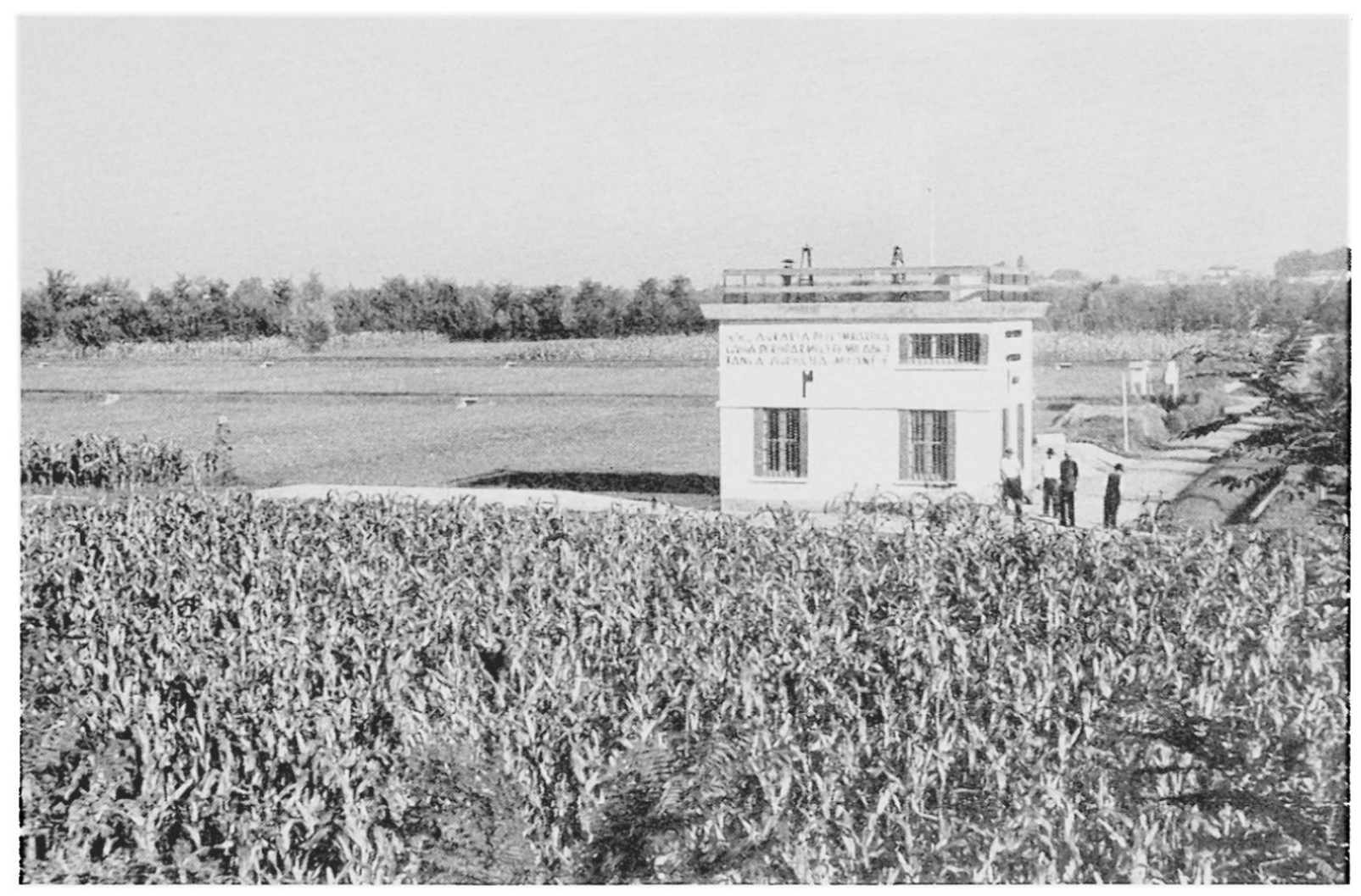

Bewässerte Felder am Villoresikanal bei Mailand

Produktionsprozesses des menschlichen Lebens Produktions- und nicht nur Verkehrsmittel sind. Die Betrachtungen haben zu zeigen versucht, daß das Netz der Beziehungen zwischen Naturgrundlage, Technik und Wirtschaft, sozialer und staatlicher Organisation bis zu den höchsten Formen der Ideologie und des Geisteslebens nirgends besser überblickbar ist als am Beispiel großer Bewässerungskulturen, wobei auch große internationale und weltgeschichtliche Beziehungen zu berücksichtigen sind. Dieser Gegenstand hat deshalb für die Geographie auch allgemein-methodische Bedeutung. Es wäre zu wünschen, daß ihm deshalb auch die künftigen Schweizerschulatlanten etwas eingehenderen Raum bieten.

\section{B E N ÜTZTE QUELLEN}

Bernhard H.: Die landbauliche Wasserwirtschaft Italiens. Bern 1919 - Le Irrigazione in Italia. 2. Auf. Rom 1931 - Ministero delle Finanze: Il gran Canale Cavour ed i minori canali demaniali d'irrigazione 1922 - 1927. Rom 1928 - Ministero delle Finanze: Il Canale Elena. Rom 19.55 Zanaboni L.: Il Canale Regina Elena (Artikel in der "Rivista del Catasto Tecnici Erariali", Nr. 5 - 6) Rom 1956 - L'impianto d'Irrigazione di Villaregia. Turin 1928 - L'Associazione d'Irrigazione dell'Agro all'Ovest del Sesia in Vercelli 1853 - 1953 - L'Associazione Irrigazione Est Sesia: Una cinquantesimale Aspirazione, un Venticinquennio di Vita 1872-1922, 1923-1947. Novara 1948 - Associazione Irrigazione Est Sesia: Gründung, Entwicklung, Ziele. Novara 1958 - Canal.INI G.: Il problema del riordino delle utenze irrigua; aspetti tecnici e giuridici. Novara 1947 - Canale Villoresi. Mailand 1958 - BERTÉ M.: La sperimentazione irrigua nel comprensorio del canale Villoresi nel Sessennio 1936 - 1941. Mailand 1942 - La Stazione Sperimentale di Risicoltura e delle coltivazioni irrigue. Vercelli 1953 - Piacco R.: La coltivazione del riso in Italia. Mailand 1955.

\section{IRRIGAZIONE DELLA PIANURA PADANA}

In Italia ben quattro-quinti dei terreni irrigati si trovano nella pianura padana; un terzo in Piemonte e un terzo in Lombardia. Lo studio si limita alla regione della pianura padana tra la Dora Baltea e l'Adda, che è attraversata dal più grande affluente del Pò, il Ticino. In questa parte la pianura mostra una rete densa e multiforme di canali, che è sorta nel corso di mille anni e ancora oggi viene allargata e migliorata. Si tratta di parecchie centinaia di canali di 
una lunghezza totale di circa $10000 \mathrm{~km}$. All'ovest del Ticino i più importanti sono: il Canale Cavour; il Canale Elena, inaugurato nel 1954, che conduce a quello l'acqua del Ticino; e tutti gli altri canali statali piemontesi (Canali Demaniali). All'est del Ticino i canali: Villoresi, Naviglio Grande, Martesana, Pavia, Muzza e quello industriale di Vizzola. I laghi alpini sono stivati da dighe regolatrici per aumentare la massa d'acqua da mettere a disposizione. I più grandi canali scorrono parelleli alle Alpi e al $\mathrm{Po}$, da un affluente all'altro, cosicchè la parte della pianura verso il Po può essere quasi completamente irrigata. Là, dove i canali passano su terreno ciottoloso, devono essere murati per impedire grandi perdite d'acqua. Sopratutti, il canale Villoresi, che scorre nell'asciutta pianura padana al Nord di Milano, è uno di questi. Gli interessati all'irrigazione sono, nella zona di cui abbiamo parlato, riuniti in tre grandi società (Associazione d'irrigazione Ovest e Est Sesia, Consorzio Villoresi), che provvedono alla ripartizione dell'acqua, al mantenimento e al miglioramento dei canali. Anche per le acque del 'Ticino e dell'Adda sono stati creati speciali Consorzi. Le maggiori colture d'irrigazione sono le risaie e le marcite.

\section{MANNHEIM - LUDWIGSHAFEN}

\section{Gerhard Ammann}

Wer in der Oberrheinischen Tiefebene nordwärts fährt, wird häufig und zu jeder Jahreszeit nördlich von Karlsruhe, etwa auf der Höhe von Speyer, cine zunehmende Trübung der Luft feststellen können. Es ist dies das erste Anzeichen, daß man sich einem hochindustrialisierten Gebiet nähert, nämlich dem Raume Mannheim-Ludwigshafen. Betreten wir dann eine der beiden Städte, so stellen wir nicht nur nebelartigen, oft sehr dichten Dunst fest, sondern auch einen beißenden Geruch, der auf Abgase chemischer Industrie schließen läßt. Es liegt eine Industriezusammenballung vor uns, wie sie Süddeutschland in dieser Größe und Ausprägung sonst nicht mehr kennt. Ihre Entstehung, Entwicklung und heutige Bedeutung soll im Folgenden darzustellen versucht werden.

\section{LAGE，NATÜRLICHE UND HISTORISCHE VORAUSSETZUNGEN}

Der nördliche Teil der Oberrheinischen Tiefebene, in dem Mannheim und Ludwigshafen liegen, wird im $\mathrm{W}$ durch die Haardt oder den Pfälzer Wald und im E durch den Odenwald (N) und den Schwarzwald (S) begrenzt. Zwischen Odenwald und Schwarzwald schiebt sich ein $50 \mathrm{~km}$ breites, sanft nach $\mathrm{E}$ ansteigendes Hügelland ein, der Kraichgau, auch Kraichgaulücke genannt. Sie stellt die ideale Pforte für den Verkehr von $E$ in die Tiefebene oder von $W$ nach dem mittleren Neckar, dann nach Franken und Bayern dar.

Die beiden Städte liegen nur noch $95 \mathrm{~m}$ ü. M. an und gegenüber der Neckarmündung, zu beiden Seiten des Rheines. Sie befinden sich nicht innerhalb der überschwemmungssicheren Zone auf den Hochufern (mehrere Meter hohe Erosionsstufe), sondern außerhalb derselben in der Flußniederung (Rheinaue). Zwischen den beiden Hochufern mäandrierte der Rhein sehr stark und überschwemmte bei Hochwasser regelmäßig große Gebiete. Auch der Neckar veränderte seinen Lauf in historischer Zeit noch wesentlich. So nimmt man an, daß die Neckarmündung im Frühmittelalter noch südlich der heutigen Stadt Mannheim lag.

Da im Mittelalter in der Rheinaue wegen dieser dauernden Überschwemmungsgefahr Siedlungen von Bedeutung nicht entstehen konnten, entwickelten sich auch Mannheim und Ludwigshafen erst in der Neuzeit.

Im Raume Mannheim-Ludwigshafen waren Speyer und Worms die wichtigsten Städte links des Rheines. Sie liegen auf dem Hochufer, das dort ursprünglich bis hart an den Fluß vorstieß. Zwischen den erwähnten Städten wurde der Rheinlauf von den Neckarfluten stark nach W gedrängt, und das Hochufer hat sich entsprechend schwach 